\title{
NON-COMPACT SYMMETRIES, EXTENDED SUPERGRAVITIES AND THE WHEELER-DEWITT EQUATION
}

\author{
H. NICOLAI \\ II Institute for Theoretical Physics, University of Hamburg, Luruper Chaussee 149, D-2000 Hamburg 50, FRG
}

Received 4 October 1989; revised manuscript received 23 November 1989

\begin{abstract}
The algebra of local and rigid symmetries of $N=16$ supergravity in three and two dimensions is considered. The conserved currents of the non-compact rigid $\mathrm{E}_{8}$ and $\mathrm{E}_{9}$ symmetries are explicitly given. On the physical states, the associated charges are found to commute with all local symmetries and, in particular, with the Whecler-DeWitt hamiltonian. The solutions of the latter are therefore infinitely degenerate. This analysis sheds some new light on the significance of non-compact symmetries in extended supergravities. Furthermore, $I$ argue that $E_{q}$ plays the role of a "solution-generating group" for the Wheeler-DeWitt equation of $N=16$ supergravity in two dimensions, which should thus be completely integrable.
\end{abstract}

Among the attempts to quantize gravity, the Wheeler-DeWitt (WDW) equation is perhaps the most promising with respect to a possible "pre-geometric" formulation of quantum gravity [1]. Nonetheless, it has so far not proved as fruitful in promoting our understanding of quantum gravity as onc might have hoped. Even if one puts aside the divergence problems of pure quantum gravity, solutions are cither rather formal and thus difficult to interpret [2], or can be obtained only under very restricted circumstances (such as the assumption that the evolution of the universe can be understood in terms of its radius and, say, a spatially constant scalar field, see e.g. ref. [3]). There is widespread agreement that these difficulties cannot be overcome without bringing additional elements into the picture, for instance by embedding gravity into superstring theory. In this paper, the WDW equation is considered in the context of three- and two-dimensional extended supergravities, and for the sake of definiteness, I will restrict attention to the maximally extended $N=16$ theories. The main emphasis here will be placed on the algebraic structure of local and rigid symmetries and their possible interpretation. A crucial difference between simple and extended supergravities is the presence of non-compact rigid symmetries in the extended theories [4]. As will be shown below, these commute with all local symmetries on the physical subspace defined by the constraints. In particular, they commute with the WDW hamiltonian, thus implying an infinite degeneracy of the WDW eigenstates. Of course, this presupposes the absence of negative norm states in the physical Hilbert space, which is far from evident as it is already quite difficult to construct a suitable Hilbert space of WDW wave functionals [2]. Assuming that this problem can be resolved, it follows that, if one solution of the WDW hamiltonian is known, infinitcly many others can be generated from it through symmetry transformations. This is also true for extended supcrgravitics in higher dimensions.

Another new element is the proposal to study the WDW equation for supergravity theories in less than four dimensions, where gravity (or pure supergravity) has no physical degrees of freedom. This may appear rather non-sensical at first sight since the WDW equation is then a statement about "supermatter" rather than gravity. Nonetheless, the "supermatter" here just corresponds to the transverse degrees of freedom of the higher-dimensional supergravity from which it originates. Consequently, one may learn something about the hidden symmetries of extended supergravities in higher dimensions by integrating the WDW equation in lower dimensions. Below three dimensions, the hidden symmetries become infinite dimensional. The $N=16$ theory in two dimensions was 
shown in ref. [5] to be integrable in the sense that there exists a linear system for its equations of motion. As will be shown below, its invariance under rigid $E_{9}$ is also reflected in the existence of a conserved $E_{9}$ current. The eigenstates of the WDW hamiltonian now form representations of the algebra gencrated by the associated charges, and this should lead to an even larger degeneracy of the WDW cigenstates. It is then an almost obvious conjecture that the $N=16$ theory is quantum-integrable, with $\mathrm{E}_{9}$ (or some descendant thereof) playing the role of a "solution-generating group" for the WDW cquation.

The prominent role of non-compact symmetries in disentangling the non-linearities of extended supergravity is well known, yet beyond this technical aspect, few attempts have been made to understand their physical significance. In refs. [6,7], unitary supermultiplets of "one-particle states" and their possible phenomenological manifestations were studied. In contrast, we are concerned here with the action of the non-compact symmetries on the physical states. These must be solutions of the WDW equation, and should rather be thought of as "multi-particle states" which encode all the information about the theory, even in situations where no asymptotic states can be defined (c.g. in the presence of non-trivial gravitational excitations). Whether the "onc-particle states" of ref. [6] correspond to observable states in any sense remains, of course, a difficult dynamical problem. A proper understanding of this issue and of the true physical significance of the non-compact symmetries is intimately tied to the interpretation of the WDW equation itself and beyond the scope of this paper.

Three-dimensional supergravity was constructed in ref. [8], and I will here only bricfly summarize the essential and pertinent results. The conventions and notation are the same as in ref. [8], cxcept for some minor additions to be indicated below. The physical fields of the three-dimensional $N=16$ theory constitute an irreducible $N=16$ supermultiplet with 128 bosons and 128 fermions transforming as incquivalent fundamental spinor representations of $\mathrm{SO}(16)$. In addition, the theory contains a dreibcin $V_{\mu}^{a}$ and 16 gravitino fields $\psi_{\mu}^{\prime}$, which do not correspond to physical degrees of freedom. The actual construction of the theory is greatly facilitated by exploiting the rigid non-compact $E_{8}$ invariance of the theory. As in ref. [8], the 248 generators of $E_{8}$ are split into 120 gencrators $X^{I J}=-X^{J I}$ and 128 generators $Y^{A}$ in accordance with the decomposition $\mathbf{2 4 8} \rightarrow \mathbf{1 2 0} \oplus \mathbf{1 2 8}$ of $\mathrm{E}_{8}$ under its $\mathrm{SO}(16)$ subgroup, where the indices $I, J$, $\ldots=1, \ldots, 16$ and $A, B, \ldots=1, \ldots, 128$ (or $\dot{A}, \dot{B}, \ldots=1$, ..., 128) label the vector representation and the fundamental spinor (or conjugate spinor) representation of $\mathrm{SO}(16)$, respectively. The rigid $\mathrm{E}_{8}$ invariance of the theory can be linearly realized in the usual manner by introducing a local $\mathrm{SO}(16)$ invariance. Consequently, the scalars $\mathscr{V}(x)$ are properly described as clements of the coset space $\mathrm{E}_{8(+8) /} \mathrm{SO}(16)$, and the "composite" $\mathrm{SO}(16)$ gauge field $Q_{\mu}^{I J}(x)$ is obtained from the $E_{8}$ Lie algcbra decomposition

$\mathscr{V}^{-1} \partial_{\mu} \mathscr{V}=\frac{1}{2} Q_{\mu}^{I J} X^{I J}+P_{\mu}^{A} Y^{A}$.

The supersymmetry transformations are given by

$\delta V_{\mu}^{a}=\mathrm{i} \bar{\epsilon}^{I} \gamma^{a} \psi_{\mu}^{I}$,

$\delta \psi_{\mu}^{I}=\mathrm{D}_{\mu} \epsilon^{I}-\frac{1}{4} \mathrm{i} \gamma^{\nu} \epsilon^{J} \bar{\chi} \Gamma^{I J} \gamma_{\mu \nu} \chi$,

$\delta \chi^{\dot{A}}=\frac{1}{2} \mathrm{i} \gamma^{\mu} \epsilon^{\prime} \hat{P}_{\mu}^{A} \Gamma_{A \dot{A}}^{\prime}$,

$\mathscr{V}^{-1} \delta \mathscr{H}=\bar{\epsilon}^{I} \chi^{\dot{A}} \Gamma_{A \dot{A}}^{I} Y^{A}$,

where $\hat{P}_{\mu}^{A}$ denotes the supercovariant extension of $P_{\mu}^{A}$ (see ref. [8] for details). The derivative $\mathrm{D}_{\mu}$ is covariant with respect to all local symmetries; for instance,

$\mathrm{D}_{\mu} \epsilon^{\prime}=\left(\partial_{\mu}+\frac{1}{4} \omega_{\mu a b} \gamma^{a b}\right) \epsilon^{I}+Q_{\mu}^{I J} \epsilon^{J}$.

The equations of motion read

$$
\begin{aligned}
& \mathrm{D}^{\mu}\left(P_{\mu}^{A}-\bar{\psi}_{\nu}^{I} \gamma_{\mu} \gamma^{\nu} \chi^{\dot{A}} \Gamma_{A \dot{A}}^{\prime}\right) \\
& \quad=\frac{1}{2} \epsilon^{\mu \nu \nu} \bar{\psi}_{\mu \nu}^{I} \psi_{\nu}^{J} \Gamma_{A B}^{I J} P_{\rho}^{B}+\frac{1}{8} \mathrm{i} \bar{\chi} \gamma^{\mu} \Gamma^{I J} \chi \Gamma_{A B}^{I J} P_{\mu}^{B}, \\
& \epsilon^{\mu \alpha \nu} \mathrm{D}_{\nu} \widehat{\psi}_{\rho}^{I}=\frac{1}{2} \gamma^{\nu} \gamma^{\mu} \chi^{A} \hat{P}_{\nu}^{A} \Gamma_{A \dot{A}}^{I},
\end{aligned}
$$

where the hat indicates the supercovariant curl, and

$$
\begin{aligned}
& -\mathrm{i} \gamma^{\mu} \mathrm{D}_{\mu} \chi^{\dot{A}}=\frac{1}{2} \gamma^{\nu} \gamma^{\mu} \psi_{\nu}^{l} \hat{P}_{\mu}^{A} \Gamma_{A \dot{A}}^{L}-\frac{1}{2}(\bar{\chi} \chi) \chi^{\dot{A}} \\
& \quad+\frac{1}{24}\left(\bar{\gamma}^{\mu} \Gamma^{J} \chi_{\mathcal{L}}\right) \Gamma_{\dot{A} \dot{B}}^{L} \gamma_{\mu} \chi^{\dot{B}} .
\end{aligned}
$$

(I omit Einstein's equation as it will not be needed.)

The $\mathrm{E}_{8}$ invariance of $N=16$ supergravity implies the existence of an associated conserved current. To construct it, we proceed from the known form of this current in non-lincar $\sigma$-models, which is $\sim \mathscr{V} P_{\mu} \mathscr{V}^{-1}$ $[9,10]$. Requiring that the corresponding current contain this piece in the limit of vanishing fermion ficlds, one finds 


$$
\begin{aligned}
J^{\mu} & =\mathscr{\gamma}^{\mu}\left[\left(P^{\mu A}-\bar{\psi}_{\nu}^{I} \gamma^{\mu} \gamma^{\nu} \chi^{\dot{A}} \Gamma_{A \dot{A}}^{I}\right) Y^{A}\right. \\
& \left.+\left(\epsilon^{\mu \nu \rho} \bar{\psi}_{\nu}^{I} \psi_{\rho}^{J}+\frac{1}{4} \mathrm{i} \bar{\gamma} \gamma^{\mu} \Gamma^{I J} \chi\right) X^{I J}\right] \mathscr{\nu}-1 .
\end{aligned}
$$

To verify that this current is indeed conserved [i.e. $\partial_{\mu}\left(V J^{\mu}\right)=0$, where $\left.V \equiv \operatorname{det} V_{\mu}^{a}\right]$, one has to make use of ( 1 ) and the equations of motion given above. Note that the expression for the conserved current contains no quartic or higher order terms in the fermions. This is in contrast with the expressions for the WDW hamiltonian and the supersymmetry gencrators. The absence of higher order fermionic terms in the $E_{8}$ current is, of course, in accord with one's expectation that the non-compact symmetry acts linearly on the fermions. The associatcd $\mathrm{E}_{8}$ charges

$2=\int V J^{0} \mathrm{~d}^{2} x=\frac{1}{2} 2^{I J} X^{I J}+2^{A} Y^{A}$

are conserved if the fields satisfy their respective equations of motion and vanish at spatial infinity or are appropriately periodic.

From its definition, the invariance of 2 under general coordinate transformations in two dimensions as well as under local SO (16) is manifest. Inserting the supersymmetry variations given above and making use of the equations of motion once more, it is not difficult to show that (taking into account higher order fermionic terms)

$\delta\left(V J^{\mu}\right)=\partial_{\nu}\left(V M^{\nu \mu}\right)$,

where

$M^{\mu \nu}=\mathscr{Y}\left(\bar{\epsilon}^{I} \gamma^{\mu \nu} \chi^{\dot{A}} \Gamma_{A \dot{A}}^{J} Y^{A}+2 \epsilon^{\mu \nu \rho} \bar{\psi}_{\rho}^{I} \epsilon^{J} X^{I J}\right) \mathscr{F}^{-1}$.

Therefore, the $E_{8}$ charges are supersymmetric (i.c. $\delta$. $=0$ ) under the same conditions that ensure their conservation.

The generalization of these results to higher dimensions is very straightforward, requiring little more than notational changes. Even if the rigid symmetry is not an invariance of the lagrangian but only of the cquations of motion, the existence of an associated conserved current can be ascertained on very general grounds [10]. To extend the above considerations to two dimensions is, however, more difficult, mainly because the symmetry becomes infinite-dimensional in this case (a general discussion of bosonic non-linear $\sigma$-model in flat space can be found in ref. [9]).
From refs. [ 5,11 ], we expect that, for $N=16$ supergravity, the $\mathrm{E}_{8}$ symmetry is enlarged to its affine cxtension $E_{9}$, at least as far as the cxistence of conserved currents is concerned. This is indeed the case, as I will now demonstrate. The fundamental field of $N=16$ supergravity in two dimensions is the matrix $\hat{\mathscr{V}}$, which depends not only on the space-time coordinates, but also on a spectral parameter $t$ [5]. This matrix contains all the on-shell information about the theory. Employing the notation, conventions and gauge conditions of ref. [11], the conserved $E_{9}$ current of $d=2, N=16$ supergravity can be parametrized as follows:

$$
\begin{aligned}
\rho \hat{I}^{\mu} & =\rho K(t) \hat{\mathscr{\gamma}}(t)\left\{Y ^ { A } \left\{b_{1}(t) P^{\mu}+\tilde{b}_{1}(t) \epsilon^{\mu \nu} P_{\nu}^{A}\right.\right. \\
& \left.+\Gamma_{A \dot{A}}^{I}\left[\mathrm{i} c_{3}(t) \bar{\psi}_{2}^{I} \gamma^{\mu} \chi^{\dot{A}}+\mathrm{i} \tilde{c}_{3}(t) \bar{\psi}_{2}^{I} \gamma^{3} \gamma^{\mu} \chi^{\dot{A}}\right]\right\} \\
& +\frac{1}{2} X^{I J}\left[c_{1}(t)\left(\mathrm{i} \bar{\gamma} \gamma^{\mu} \Gamma^{I J} \gamma+8 \bar{\psi}_{2}^{I} \gamma^{3} \gamma^{\mu} \psi^{J}\right)\right. \\
& +\tilde{c}_{1}(t)\left(\mathrm{i} \bar{\gamma}^{3} \gamma^{\mu} \Gamma^{I J} \chi+8 \bar{\psi}_{2}^{I} \gamma^{\mu} \psi^{J}\right) \\
& \left.\left.+\mathrm{i} c_{2}(t) \bar{\psi}_{2}^{I} \gamma^{\mu} \psi_{2}^{J}+\mathrm{i} \tilde{c}_{2}(t) \bar{\psi}_{2}^{I} \gamma^{3} \gamma^{\mu} \psi_{2}^{J}\right]\right\} \hat{\psi}^{-1}(t) .
\end{aligned}
$$

As explained in refs. [5.11], the scalar field $\rho$ is a remnant of the dreibcin $V_{\mu}^{a}$ and satisfies the cquation $\partial^{\mu} \partial_{\mu} \rho=0$. The dependence on the conformal factor $\lambda$ has bcen absorbed through a rescaling of the fields. The various coefficient functions appearing in (14) depend on the spectral parameter $t$ and are defined by

$$
\begin{aligned}
& b_{1}(t)=\frac{1+t^{2}}{1-t^{2}}, \quad \tilde{b}_{1}(t)=\frac{2 t}{1-t^{2}} \\
& c_{1}(t)=\frac{1}{2} \frac{1+6 t^{2}+t^{4}}{\left(1-t^{2}\right)^{2}}, \quad \tilde{c}_{1}(t)=2 \frac{t\left(1+t^{2}\right)}{\left(1-t^{2}\right)^{2}} \\
& c_{2}(t)=-16 \frac{t^{2}\left(6+20 t^{2}+6 t^{4}\right)}{\left(1-t^{2}\right)^{4}} \\
& \tilde{c}_{2}(t)=-16 \frac{t\left(1+t^{2}\right)\left(1+14 t^{2}+t^{4}\right)}{\left(1-t^{2}\right)^{4}} \\
& c_{3}(t)=8 \frac{t\left(1+4 t^{2}+t^{4}\right)}{\left(1-t^{2}\right)^{3}} \\
& \tilde{c}_{3}(t)=\frac{\left(1+t^{2}\right)\left(1+22 t^{2}+t^{4}\right)}{\left(1-t^{2}\right)^{3}}
\end{aligned}
$$

It is one of the peculiar features of the two-dimensional integrable modcls, which are descendants of (possibly matter-coupled) gravity theories in higher 
dimensions, that the spectral parameter depends nontrivially on the space-time coordinates [12]. More precisely, we have $t=t(x, w)$, where $w$ is the free integration constant in the equation defining $t$ :

$t p^{-1} \partial_{\mu} \rho=\frac{1+t^{2}}{1-t^{2}} \partial_{\mu \mu} t-\frac{2 t}{1-t^{2}} \epsilon_{\mu \nu} \partial^{\nu} t$.

The overall coefficient in (14) contains the factor $K$, which is a non-local function of the spectral parameter $t$, and is defined by $\#$

$K^{-1} \partial_{\mu} K=\frac{4}{1-t^{2}} \epsilon_{\mu \nu} \partial^{\nu} t$.

The integrability of this differential equation follows from

$\partial^{\mu}\left(\frac{1}{1-t^{2}} \partial_{\mu} t\right)=0$

which itself is a consequence of (16). As before, current conservation, i.e. $\partial_{\mu}\left(\rho \hat{J}^{\mu}\right)=0$, is implied by the equations of motion, at least up to and including terms quadratic in the fermionic fields (the details of this somewhat lengthy calculation will be given elsewhere). As for higher order fermionic contributions, I have so far only verified the consistency of the $(\bar{\gamma} \chi)^{2}$ sector. Note that the $\mathrm{E}_{8}$ current is re-obtained for $t=0$. The lengthy expression (14) can be considerably simplified by use of relations between the coefficients appearing in the linear system [11] and in (15), such as

$\frac{\mathrm{d}}{\mathrm{d} t} a_{1}(t)=\frac{2}{1-t^{2}} \tilde{c}_{1}(t)$,

$\frac{\mathrm{d}}{\mathrm{d} t} \tilde{a}_{1}(t)=\frac{2}{1-t^{2}} c_{1}(t)$, etc.

where $a_{1}(t)$ and $\tilde{a}_{1}(t)$ are defined by $[5,11]$

$a_{1}(t)=\frac{2 t^{2}}{\left(1-t^{2}\right)^{2}}, \quad \tilde{a}_{1}(t)=\frac{t\left(1+t^{2}\right)}{\left(1-t^{2}\right)^{2}}$.

Furthermore, the following formula:

\#1 If $t$ depends on only one of the light-cone coordinates $x^{+}$or $x^{-}, K$ becomes local. Explicitly, one finds $K\left(x^{+}\right)=[(1-t) /$ $(1+t)]^{2}$ and $K\left(x^{-}\right)=[(1+t) /(1-t)]^{2}$. Observe that in a flat space theory, where $t$ does not depend on $x$, we would have $K=1$. $\left[\frac{\partial}{\partial t}, \partial_{\mu}\right] f=\left(t^{-1} \partial_{\mu} t+\frac{2}{1-t^{2}} \epsilon_{\mu \nu} \partial^{\nu} t\right) \frac{\partial f}{\partial t}$,

valid for any function $f(x, t(x, w))$, is useful. After some algebra, (14) reduces to ${ }^{\sharp 2}$

$\rho \hat{J}^{\mu}=\frac{1}{2} \epsilon^{\mu \nu} \partial_{\nu}\left(\rho K\left(1-t^{2}\right) \frac{\partial \hat{y}}{\partial t} \hat{y}^{-1}\right)$.

It is obvious from this result that the $\mathrm{E}_{9}$ charges

$$
\begin{aligned}
\hat{2}(w) & =\int \rho(x) \hat{J}^{0}(x, t(x, w)) \mathrm{d} x^{1} \\
= & \sum_{n=-\infty}^{\infty} w^{-n} \hat{2}_{n}
\end{aligned}
$$

can be represented as the difference of two boundary terms. As before, the $\mathrm{SO}(16)$ invariance of $\hat{J}^{\mu}$, and hence of $\hat{2}(w)$, is manifest. The expression (23) is also very convenient to analyze the behavior of the $E_{9}$ current under the "superconformal" transformations considered in ref. [11]. Using the result for $\hat{y}-1 \delta \hat{y}$ given there (eq. (3.12) of ref. [11]), it is not difficult to show that the variation of $\hat{2}(w)$ is again the difference of two boundary terms. To check the quartic fermionic terms, which have been ignored in this calculation, will be very tedious as the spectral parameter $t$ must also be varied [11].

Much of this analysis can be repeated in the hamiltonian framework (for the hamiltonian formulation of $N=1$ supergravity in four dimensions, see refs. $[13,14])$, and I will now bricfly indicate how to do this for the three-dimensional theory, postponing a more detailed discussion to a later paper. In particular, higher order fermionic terms will be disregarded in most of the remainder. It is convenient to make use of local $\operatorname{SO}(1,2)$ invariance to parametrize the dreibein as follows (see c.g. ref. [15]):

$V_{\mu}^{a}=\left(\begin{array}{cc}N & N^{\alpha} \\ 0 & e_{i}^{\alpha}\end{array}\right)$

where $i, j, \ldots=1,2$ and $\alpha, \beta, \ldots=1,2$ are curved and flat spatial indices, respectively, and $e_{i}^{\alpha}$ is the spatial zweibein; I will also use the positive definite spatial metric $h_{i j} \equiv-e_{i}^{\alpha} e_{j \alpha}$. By means of a general coordi-

\#2 For the bosonic non-linear $\sigma$-model in flat space, this simple expression for the current is due to $M$. Niedermaier. 
nate transformation the lapse and shift functions can be taken to be $N=1$ and $N^{\alpha}=0$; then $V=e=\sqrt{h}$. For the gravitino, a convenient gauge choice is $\psi_{0}^{\prime}=0$. Before inserting these gauge choices, however, we must keep in mind that the variables $N, N^{\alpha}, \psi_{0}^{l}$ and $Q_{0}^{I J}$ do not have time derivatives in the action, and their variation thus leads to constraints. One must therefore first perform the variations and use the gauge conditions only afterwards. In this way one obtains the WDW hamiltonian (or rather hamiltonian density)

$$
\begin{aligned}
\mathscr{H} & \equiv-\frac{\delta \mathscr{L}}{\delta N}=\frac{1}{4} e R^{(2)}+4 e^{-1}\left(\Pi^{2}-\Pi^{i j} \Pi_{i j}\right) \\
& +\frac{1}{4} e\left(P_{0}^{A} P_{0}^{A}+P^{i A} P_{i}^{A}-2 \mathrm{i} \bar{\chi} \gamma^{i} \mathrm{D}_{i} \chi\right. \\
& \left.+2 \bar{\chi}^{A} \gamma^{i} \gamma^{J} \psi_{i}^{I} P_{j}^{A} \Gamma_{A \dot{A}}^{I}+\ldots\right) .
\end{aligned}
$$

Here $R^{(2)}$ denotes the spatial curvature, and the dots stand for higher order fermionic terms. $\Pi^{i j}$ is the canonical momentum associated with the spatial metric $h_{i j}$, and $\Pi \equiv h_{i j} \Pi^{i j}$. The generators of spatial coordinate transformations read

$$
\begin{aligned}
\Phi_{i} & \equiv \frac{\delta \mathscr{P}}{\delta N^{i}}=-2 \mathrm{D}^{i} \Pi_{i j}-\frac{1}{2} e \epsilon^{j k} \bar{\psi}_{j}^{I} \mathrm{D}_{i} \psi_{k}^{l} \\
& +\frac{1}{2} e\left(P_{0}^{A} P_{i}^{A}-\mathrm{i} \bar{\chi} \gamma^{0} \mathrm{D}_{i} \chi+\bar{\chi}^{\dot{A}} \gamma^{j} \gamma^{0} \psi_{j}^{I} \Gamma_{A \mathcal{A}}^{I} P_{i}^{A}\right) .
\end{aligned}
$$

Finally, the generators of space-dependent supersymmetry transformations arc given by

$$
\begin{aligned}
& \mathscr{P}^{I} \equiv-\frac{\delta \mathscr{L}}{\delta \bar{\psi}_{0}^{I}}=-e \epsilon^{i j} \widehat{\mathrm{D}_{i} \psi_{j}^{I}}+\frac{1}{2} e \gamma^{\mu} \gamma^{0} \chi^{\dot{A}} \Gamma_{A \dot{A}}^{I} \widehat{P_{\mu}^{A}} \\
& =-e \epsilon^{i j}\left[\mathscr{X}_{i}+\frac{1}{4} \omega_{i \alpha \beta} \gamma^{\alpha \beta}+2 e^{-1}\left(h_{i k} \Pi-\Pi_{i k}\right) \gamma^{k} \gamma^{0}\right] \psi_{j}^{I} \\
& +\frac{1}{2} e \Gamma_{A \dot{A}}^{\prime}\left(\chi^{\dot{A}} P_{0}^{A}-\gamma^{j} \gamma^{0} \chi^{\dot{A}} P_{j}^{A}\right)+\ldots,
\end{aligned}
$$

where the $\mathrm{SO}(16)$ covariant derivative $\mathscr{T}$, has been split off, and the spin connection has been written out explicitly in terms of its spatial part and the canonical momentum. I omit the explicit expression for the generator of spatial Lorentz transformations which is straightforward to derive.

To proceed further, we need the canonical (Dirac) brackets

$$
\left\{\psi_{i}^{I}(x), \dot{\psi}_{j}^{J}(y)\right\}=e^{-1} \epsilon_{i j} \delta^{J J} \delta^{(2)}(x-y)
$$

and

$\left\{\chi^{\dot{A}}(x), \bar{\chi}^{\dot{B}}(y)\right\}=e^{-1} \mathbf{i} \gamma^{0} \delta^{\dot{A} \dot{\beta}} \delta^{(2)}(x-y)$, where spinor indices have been suppressed and the variables $x, y$ parametrize the spacelike hypersurface (all brackets are understood to be equal time brackets ). Furthermore,

$\left\{h_{i j}(x), \Pi^{k l}(y)\right\}=\delta_{i}^{(k} \delta_{j}^{\prime)} \delta^{(2)}(x-y)$

and

$$
\begin{aligned}
& \left\{\Pi^{i j}(x), \sqrt{e} \chi^{\dot{A}}(y)\right\}=\left\{\Pi^{i j}(x), \sqrt{e} e_{\alpha}^{k} \psi_{k}^{K}(y)\right\} \\
& \quad=0 .
\end{aligned}
$$

For the matter bosons, it is also convenient to choose the variables such that they commute with the fermionic ones and with $\Pi^{i j}$. As $P_{0}^{A}$ does not, we define

$\tilde{P}_{0}^{A} \equiv e\left(P_{0}^{A}+\bar{\psi}_{i}^{I} \gamma_{0} \gamma^{i} \chi^{i} \Gamma_{A \dot{A}}^{I}\right)$,

which commutes with the fermionic fields and with $\Pi^{i j}$. Observe that use of the new quantity $\widetilde{P}_{0}^{A}$ also simplifies some of the previous expressions such as (26). The relevant brackets are now given by

$$
\begin{aligned}
& \left\{\tilde{P}_{0}^{A}(x), \mathscr{Y}(y)\right\}=-2\left(\mathscr{F}^{A}\right) \delta^{(2)}(x-y), \\
& \left\{\tilde{P}_{0}^{A}(x), \tilde{P}_{0}^{B}(y)\right\} \\
& \quad=\Gamma_{A B}^{I J}\left(\phi^{I J}+\frac{1}{4} \mathrm{i} e \bar{\chi} \bar{\gamma}^{O} \Gamma^{I J} \chi+e \epsilon^{i j} \bar{\psi}_{i}^{I} \psi_{j}^{J}\right) \\
& \quad \times \delta^{(2)}(x-y)
\end{aligned}
$$

from which all other brackets can be deduced. (34) contains the canonical generators $\phi^{I J}$ of $\mathrm{SO}(16)$ gauge transformations, which satisfy

$$
\begin{aligned}
& \left\{\phi^{l J}(x), \phi^{K L}(y)\right\} \\
& \quad=4 \delta^{[x[I I} \phi^{J] K]}(x) \delta^{(2)}(x-y) .
\end{aligned}
$$

As a useful check on these brackets, one can verify that, modulo the constraints, the equations of motion are recovered from

$$
\partial_{0} \varphi(x)=\left\{\varphi(x), \int d^{2} y \mathscr{H}(y)\right\},
$$

where $\varphi$ stands for any of the fields. Likewise, the supersymmetry transformations with time-independent parameter $\epsilon^{I}(x)$ can be obtained from

$$
\delta_{\epsilon} \varphi(x)=\left\{\varphi(x), \int \mathrm{d}^{2} y \bar{\epsilon}^{I}(y) \mathscr{S}^{\prime}(y)\right\} .
$$

There is a subtlety, however, when this formula is applied to $\widetilde{P}_{0}^{A}$, because, from $(5), \delta P_{0}^{A}=\mathrm{D}_{0}\left(\bar{\epsilon}^{I} \chi^{\dot{A}} \Gamma_{A \dot{A}}^{\prime}\right)$, 
which contains a time derivative and hence cannot be obtained without use of the equations of motion.

Given these brackets, it is now possible to investigate the algebra of local and rigid symmetrics. We are here primarily interested in those parts of the algebra involving 2. Neglecting possible contributions from higher order fermionic terms, one finds

$$
\begin{aligned}
& \left\{\mathscr{F}^{I}(x), \mathscr{Q}\right\}=-2\left(\mathscr{\mathscr { V } ^ { \prime }} X^{I J \mathscr{Y}^{-1}}\right) \mathscr{F}^{J}(x) \\
& -\left(\Gamma^{I} \Gamma^{K L}\right)_{\Lambda \mathrm{i}}\left(\mathscr{Y} Y^{A} \mathscr{V}^{-1}\right) \chi^{\dot{A}} \dot{\phi}^{K L}(x), \\
& \left\{\Phi_{i}(x), 2\right\}=\frac{1}{2} \Gamma_{A B}^{I J} P_{i}^{A}\left(\mathscr{Y}^{B} Y^{B} \mathscr{Y}^{-1}\right) \phi^{I J}(x)
\end{aligned}
$$

and

$$
\{\mathscr{H}(x), 2\}=\frac{1}{2} \Gamma_{A B}^{I J} P_{0}^{A}\left(\mathscr{W} Y^{B} \mathscr{F}^{-1}\right) \phi^{L J}(x) \text {. }
$$

This shows that, on the constraint surface $\mathscr{H}=\Phi_{i}=$ $\phi^{I J}=\mathscr{P}^{I}=0,2$ indeed commutes with all local symmetries. Commutators similar to (38) between local supcrsymmetry transformations and rigid symmetries have already been evaluated in refs. $[6,7,16]$. The connection with these results is easily cstablished by expanding $\mathscr{r}$ in terms of the scalar fields in the "unitary" gauge. The non-vanishing result of this computation was interpreted as evidence for the existence of a "spectrum generating" supergroup, with an infinite tower of new supercharges constructed out of $\mathscr{P}$ and products of the scalar fields. However, the above result suggests a different interpretation, because (38)-(40) only fail to vanish off shell. On the mass-shell, the rigid non-compact symmetries do commute with supersymmetry, and the new supercharges defined in this manner should therefore be regarded merely as generators of off-shell symmetries.

In the quantum theory, the constraints become operator constraints on the physical states of the theory. The bosonic physical state conditions are (including the WDW equation )

$$
\mathscr{H}(x) \Psi=\Phi^{i}(x) \Psi=\phi^{I J}(x) \Psi=0,
$$

where $\Psi$ is the WDW wave functional ("wave function of the universe"). I have here blithely ignored ordering problems, assuming that possible quantum divergences of the theory are sufficiently softened (it is probably here that maximal symmetry is needed). Furthermore, since the commutator of two local supersymmetry variations contains all the other symmetries with field-dependent parameters [17], onc cannot a priori exclude ordering problems and anomalies in the quantum algebra of constraints as well; again, maximal symmetry may be necessary here to ensure a safe passage to the quantum theory. Pending a morc detailed investigation of this issue, let us assume that no such complications arise for the $N=16$ theory. Then all of the above conditions are implied by

$\mathscr{P}^{I}(x) \Psi=0$.

This is the "square-root" of the WDW cquation. It is at this point that local supersymmetry is crucial; in a rigidly supersymmetric theory, such as a supersymmetric non-linear $\sigma$-model in flat space, we would also have $\{\mathscr{P}, \mathscr{P}\} \sim H$, but acting with $\mathscr{f}$ on a state would just give another state and not an equation with dynamical content. From (38)-(40), it is now obvious that 2 commutes with all local generators on the physical states. Consequently, if $\Psi$ is a solution of the WDW equation then $2 \Psi$ is another, and thus all solutions form multiplets of $E_{8}$. There exist well-developed methods to construct unitary representations of non-compact $\mathrm{E}_{8}$, see e.g. ref. [7].

While it is straightforward to verify that the $E_{8}$ charges 2 satisfy the expected commutation relations on the physical states, the algebra of the $E_{9}$ charges (23) has so far not becn evaluated. The explicit appearance of the matrix $\hat{\psi}$ in (14) implies that the higher charges will be non-local, because $\hat{V}$ - being the solution of a linear system - is given by a pathordered exponential. Even in the much simpler case of the principal chiral model in flat space, the computation of Poisson brackets of non-local charges is marred by technical complications and ambiguities [18]. In particular, one finds that the commutator of two non-local charges contains new charges made out of products of the basic charges, and that the nonlocal charges close into a Yang-Baxter type algebra rather than an affine Kăc-Moody algebra. For this reason, it is far from clear whether the generators (23) will simply close into an $E_{9}$ Kač-Moody algebra. Rather, there is the intriguing possibility that the resulting algebraic structure extends beyond $E_{9}$ and could even be related to $E_{10}$, whose realization in this context was conjectured long ago [19]. To find out, one must, of course, first come to grips with the technical problem of calculating the relevant Poisson brackets (or commutators). Finally, whatever the outcome of these calculations will be, the emerging 
algebraic structure may lead to a complete classification of the "observables" associated with the WDW equation of $N=16$ supergravity, and thus to the resolution of one of the outstanding problems of quantum gravity [2], at least in this special case.

I am grateful to I. McArthur, B. de Wit, M. Niedermaier and A. Sagnotti for stimulating discussions related to this work.

\section{References}

[1] J.A. Wheeler, in: Relativity, groups and topology, cds. C. DeWitt and B. DeWitt (Gordon and Breach, New York, 1964);

B. DeWitt, Phys. Rev. 160 (1967) 113; 162 (1967) 1195.

[2]A. Ashtekar, New perspectives in canonical gravity (Bibliopolis, 1988);

T. Jacobson and L. Smolin, Nucl. Phys. B 299 (1988) 295.

[3] S.W. Hawking, in: Relativity, groups and topology II, eds. B. DeWitt and R. Stora (North-Holland, Amsterdam, 1984).

[4] E. Cremmer, S. Ferrara and J. Scherk, Phys. Lett. B 74 (1978) 61

E. Cremmer and B. Julia, Nucl. Phys. B 159 (1979) 141.
[5] H. Nicolai, Phys. Lett. B 194 (1987) 402.

[6] J. Ellis, M.K. Gaillard and B. Zumino, Phys. Lett. B 94 (1981) 221.

[7] $M$. Günaydin, in: Unification of the fundamental interactions II, eds. J. Ellis and S. Ferrara (Plenum, New York, 1983).

[8] N. Marcus and J.H. Schwar,, Nucl. Phys. B 228 (1983) 145.

[9] M. Forger and H. Eichenherr, Commun. Math. Phys. 82 (1981) 227.

[10] M.K. Gaillard and B. Zumino, Nucl. Phys. B 193 (1981) 221.

[11] H. Nicolai and N.P. Warner, Commun. Math. Phys. 125 (1989) 384.

[12] P. Breitenlohner and D. Maison, Ann. Inst. Henri Poincaré 46 (1987) 215.

[13] S. Deser, J.H. Kay and K.S. Stelle, Phys. Rev. D 16 (1977) 2448.

[14] E.S. Fradkin and M.A. Vasiliev, Phys. Lett. B 72 (1977) 70; M. Pilati, Nucl. Phys. B 132 (1978) 138.

[15] C. Misner, K. Thorne and J.A. Whecler, Gravitation (Freeman, San Francisco, 1973).

[16] N. Marcus, A. Sagnotti and J.H. Schwarz, Nucl. Phys. B 243 (1984) 335 .

[17] P. van Nicuwenhuizen, Phys. Rep. 68 (1981) 189

[18] M. Lüscher and K. Pohlmeyer, Nucl. Phys. B 137 (1978) 46 ;

H.J. de Vega, H. Eichenherr and J.M. Maillet, Commun. Math. Phys. 92 (1984) 507.

[19] B. Julia, in: Superspace and supergravity, eds. S.W. Hawking and M. Roček (Cambridge U.P., Cambridge, 1980). 